\title{
The Tympanic Membrane: Highly Developed Smooth Muscle Arrays in the Annulus Fibrosus of Mustached Bats
}

\author{
O.W. Henson JR. ${ }^{1}$ And Miriam M. Henson ${ }^{2}$ \\ ${ }^{1}$ Department of Cell Biology and Anatomy, The University of North Carolina Chapel Hill, NC 27599, USA \\ ${ }^{2}$ Department of Surgery, Division of Otolaryngology/Head and Neck Surgery, The University of North Carolina, \\ Chapel Hill, NC 27599, USA
}

Received: 13 December 1999; Accepted: 3 March 2000; Online publication: 31 May 2000

\begin{abstract}
The annulus fibrosus tympanicus is the thickened peripheral rim of the pars tensa of the tympanic membrane. It is an area into which the connective tissue matrix of the membrane extends to attach to the tympanic bone (ring). Light microscopy, SEM, TEM and confocal microscopy were used to study the area in mustached bats Pteronotus $p$. parnellii, $P$. p p portoricensis and $P$. quadridens. In cross sections of the annulus morphologically distinct apical and basal or myovascular zones could be recognized. The apical zone had a collagenous matrix that was continuous with the pars tensa. The myovascular zone contained an extensive network of thin walled endothelial tubes and a welldeveloped array of radially arranged smooth muscle cells that filled the interval between the vessels. The muscle tissue occupied the interval between the densely collagenous "apical zone" and the bony tympanic ring and was closely associated with bundles of unmyelinated nerve fibers. The structure and arrangement of the tissue suggests a highly developed specialization for the tonic control of tension of the pars tensa and a system that potentially regulates sound transmission to the middle and inner ear.
\end{abstract}

Keywords: tympanic membrane, annulus fibrosus, smooth muscle, bats

Correspondence to: Dr. O.W. Henson Jr. • The University of North Carolina • Taylor Hall • CB \#7090 • Chapel Hill, NC 27599. Telephone: (919) 843-9334; fax: (919) 966-1856; e-mail: owh@ med.unc.edu

\section{INTRODUCTION}

In mammals, the pars tensa of the tympanic membrane typically has a middle fibrous layer with radially and circumferentially arranged collagen fibers (see Lim, 1995 for review). The peripheral rim of this matrix is a thickened area (annulus fibrosus tympanicus) where the radial fibers fan out to attach along the inner margin (sulcus tympanicus) of the tympanic bone (ring; annulus tympanicus). The annulus fibrosus characteristically has numerous blood vessels, radially arranged collagen fibers and many fibroblasts. In this report we will show that the annulus of mustached bats additionally has a well-developed system of radially arranged smooth muscle cells. These bats appear to have a specialized system for the tonic regulation of tension in the tympanic membrane.

\section{METHODS}

\section{Animals used}

The animals used in this study were mustached bats, Pteronotus parnellii parnellii, Pteronotus parnellii portoricensis and Pteronotus quadridens. Permission to collect in Jamaica was approved by the Natural Resources Conservation Authority and Ministry of Agriculture, Division of Veterinary Services of Jamaica. Permits for collection in Puerto Rico were obtained from the Natural Resources Department in Puerto Rico. The care and use of all animals was approved by the Institutional Animal Care and Use Committee at The University of North Carolina at Chapel Hill, Animal Assurance 


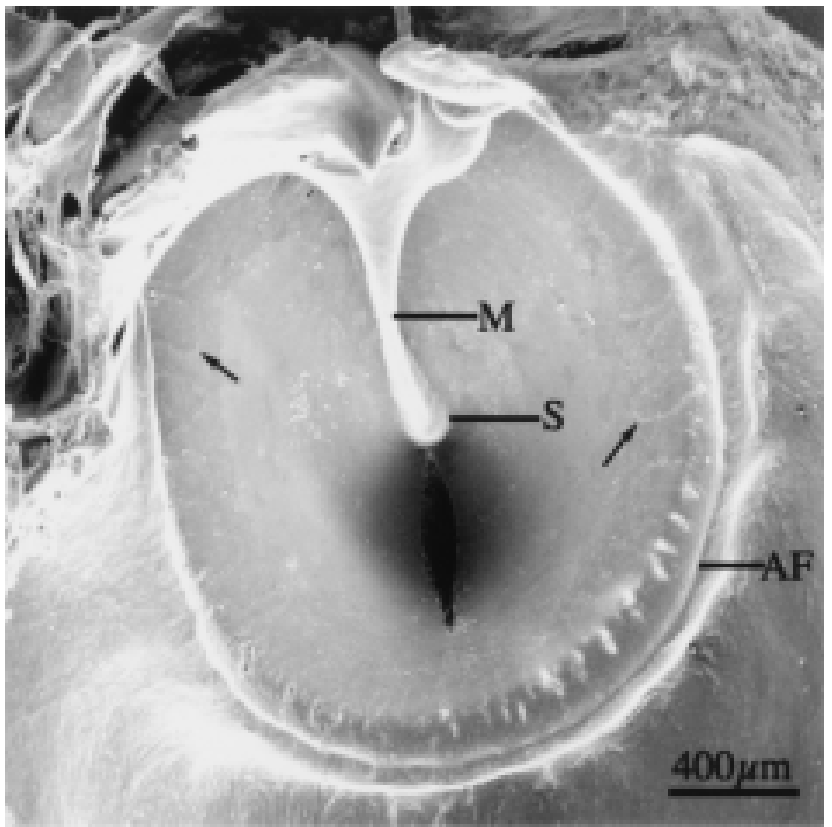

FIG. 1. SEM of internal surface of a whole mount preparation of the tympanic membrane, pars tensa (Pteronotus parnellii portoricensis). Note the annulus fibrosus (AF) and malleus (M). The spatulated tip of the manubrium (S) marks the position of the umbo. Arrows mark the position of capillary loops near the rim of the pars tensa.

Number A3410-01. TEM and SEM studies were restricted to $P$. p. portoricensis.

\section{Isolation and fixation of tissue}

The animals were anesthetized with Metofane (Pitman Moore, NJ) and killed by decapitation. In bats, the tympanic ring is a relatively loose, simple ring-like bone which serves as the attachment area for the pars tensa (Henson, 1961, 1974). Whole mount preparations of the intact ring and attached membrane were prepared by cutting off the external ear close to the tympanic membrane, then splitting the head in the midsagittal plane and shelling out the cochlea to expose the internal surface of the tympanic membrane and attached tensor tympani muscle. After further trimming all that remained was the bony ring, tympanic membrane and malleus (Fig. 1). The malleus is firmly fused to the tympanic bone so the preparation could be treated as a single piece of tissue and immersed in fixative.

\section{Confocal microscopy}

Bouin's fixative was used for tissue intended for confocal microscopy. Tissue processing and final staining with Mallory trichrome followed the method of Baird and Henson (1960). The only modification of the method was that decalcification was accomplished with EDTA in a microwave oven according to the method of Madden and Henson (1997). Thick (75 $\mu \mathrm{m})$ sections were examined with a Zeiss LSM 210XX confocal laser scanning microscope using the $514 \mathrm{~nm}$ line of an argon ion laser and an emission 575-645 nm band pass filter. The wavelengths employed resulted in excitation of the aniline blue in the trichrome stain. Both $60 \mathrm{X}$ oil immersion and 40X dry objectives were used. With this system we could focus deeply into the tissue and collect sequential fluorescent images at controlled intervals. In cross sections of the annulus, individual muscle fibers could be followed over their entire length and in tangential sections the blood vessels and their interconnections could be traced.

\section{Light and transmission electron microscopy}

For both light and transmission electron microscopy, the fixative consisted of $2 \%$ paraformaldehyde and $2.5 \%$ glutaraldehyde in $0.15 \mathrm{M}$ sodium phosphate buffer, $\mathrm{pH}$ 7.4. Tissue was fixed for 12-24 hours in the cold $\left(4^{\circ} \mathrm{C}\right)$ and decalcified in $0.1 \mathrm{M}$ EDTA in $0.15 \mathrm{M}$ sodium phosphate buffer in a microwave oven (Madden and Henson, 1997). It was then osmicated, dehydrated, embedded in epoxy resin, sectioned and stained according to standard procedures.

For light microscopy tissue was sectioned at $1 \mu \mathrm{m}$. For TEM, specimens were sectioned at $70 \mathrm{~nm}$ and viewed on a LEO EM910 transmission electron microscope (LEO Electron Microscopy, Inc., Thornwood, $\mathrm{NY})$ at an accelerating voltage of $60 \mathrm{kV}$.

\section{Scanning electron microscopy}

Tissue preparation consisted of fixation and decalcification as described above for light and TEM. It was then critical point dried, mounted on stubs and coated with gold-palladium. The tissue was viewed with a LEO S200 scanning electron microscope.

\section{RESULTS}

\section{Structure of the pars tensa}

The pars tensa was studied only to the extent that its basic composition could be established and compared with that known for common laboratory mammals. It was similar to other animals in that: 1) it was coneshaped with the deepest part, the umbo, corresponding in position to the spatulated tip of the manubrium (Fig. 1); 2) the fibrous stratum consisted of an outer layer of radially oriented fibers and an inner circular layer (Fig. 2); 3) the external surface was covered by two layers of keratinocytes and was continuous with the lining of the external acoustic meatus (Fig. 3); 4) the internal surface was covered with a mucosal lining 

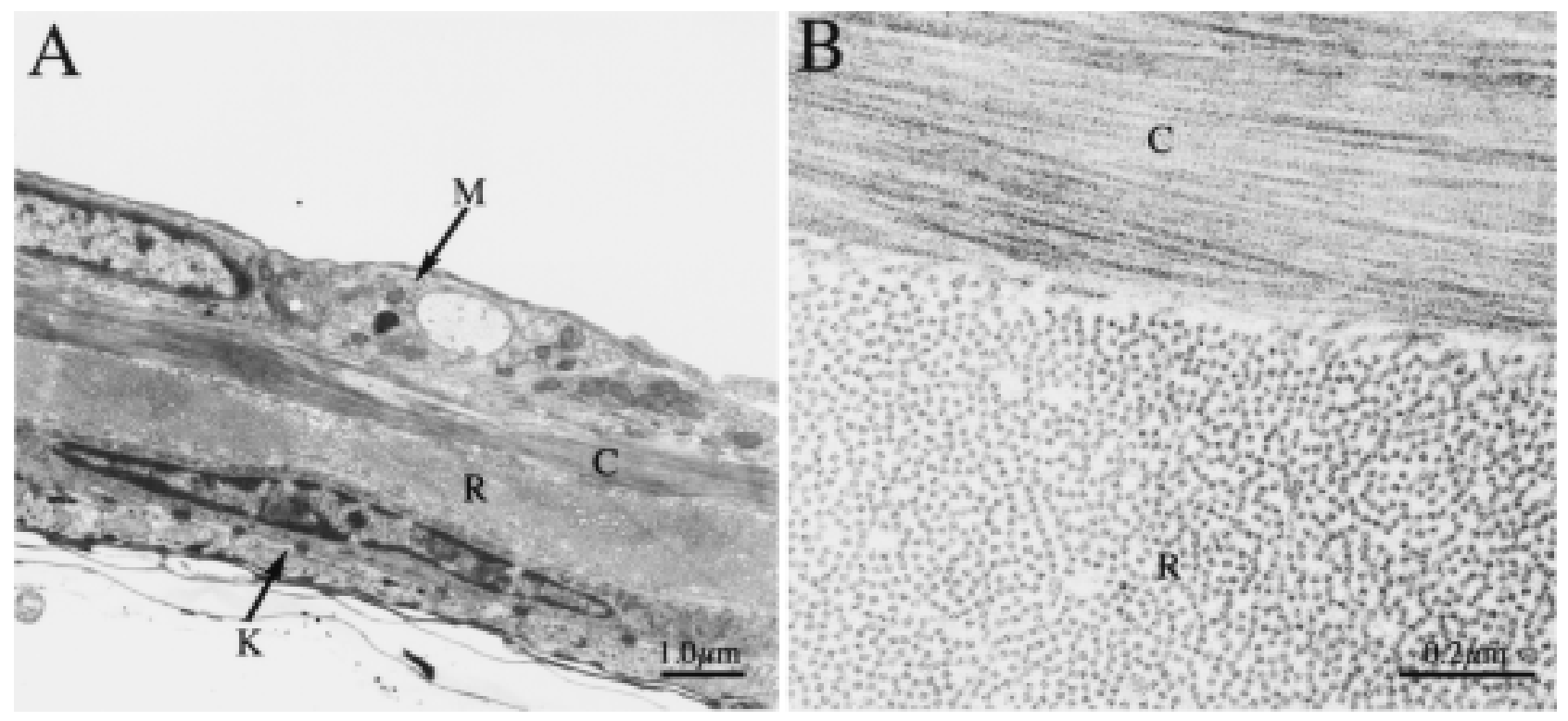

FIG. 2. TEM of the pars tensa of the tympanic membrane (P. p. portoricensis). A illustrates the thinness of the membrane. Note the two layers of keratinocytes $(K)$ lining the external surface of the membrane and the mucosal layer $(M)$ on the internal surface. Between these cell layers is the fibrous stratum, shown in high magnification in B. This consists of outer radial (R) and inner circular (C) layers of collagen.

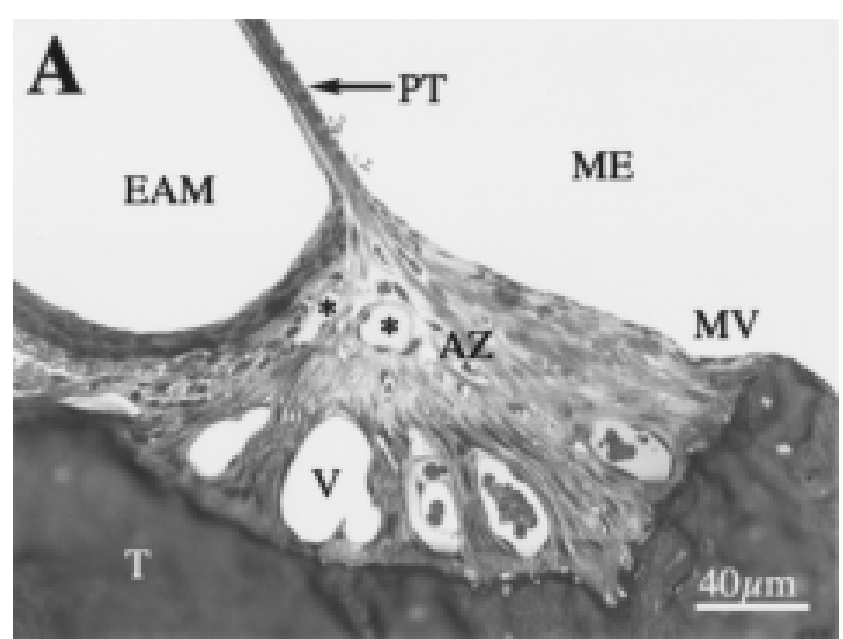

FIG. 3. Light micrographs of cross-sections of the tympanic bone and attached annulus fibrosus (P. p. portoricensis). A is a low magnification showing the pars tensa (PT) and the position of the apical zone (AZ) and myovascular zone (MV). In the AZ, blood vessels are marked with asterisks and are surrounded by collagen; the MV

continuous with that of the middle ear cavity (Figs. 2, 3 ); and 5) the radially oriented fibers attached to the manubrium and extended peripherally into the annulus fibrosus (Fig. 3). One notable difference between the tympanic membrane in mustached bats and most other mammals is its small size (ca. $1.6 \mathrm{~mm}$ in diameter and only 4.5-5.6 $\mu \mathrm{m}$ thick). Even though it is very thin, the basic layers are distinct (Fig. 2). It is interesting to note that the thickness of the membrane is approximately the diameter of a red blood cell; no vessels were seen in TEM micrographs in the

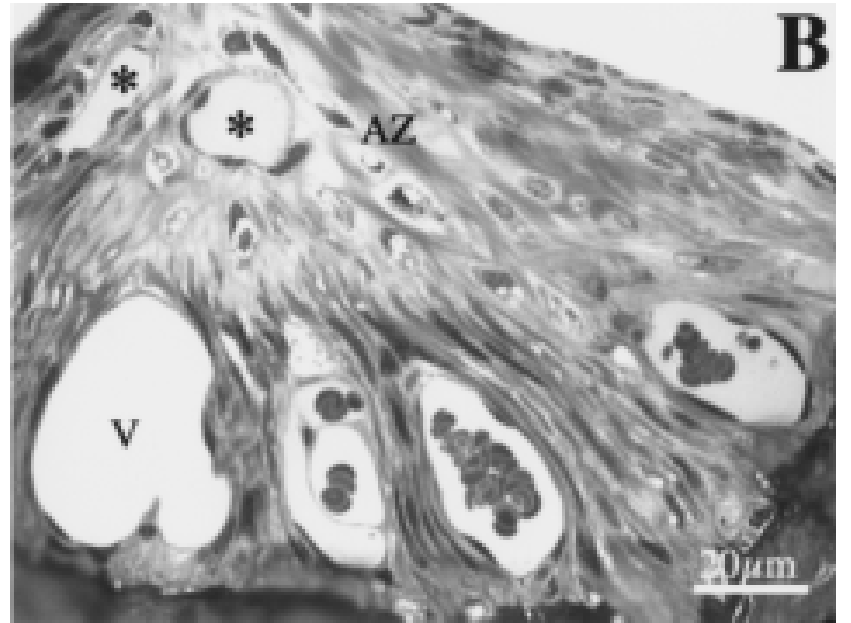

contains large vessels $(\mathrm{V})$ and interposed smooth muscle cells. EAM, external acoustic meatus; ME, middle ear cavity; T, tympanic bone. $B$ is a higher magnification of the same section and better illustrates the orientation, extent and light microscopic appearance of the smooth muscle.

areas examined. With SEM, however, there were indications of capillary loops near the rim of the pars tensa (Fig. 1) and these were confluent with capillaries in the peripheral margin of annulus fibrosus.

The annulus fibrosus

The annulus is triangular in cross-section, with the apex pointing toward the pars tensa (Fig. 3). In light micrographs it can be seen that the apical half (zone) has many small vessels, mostly capillaries, scattered 


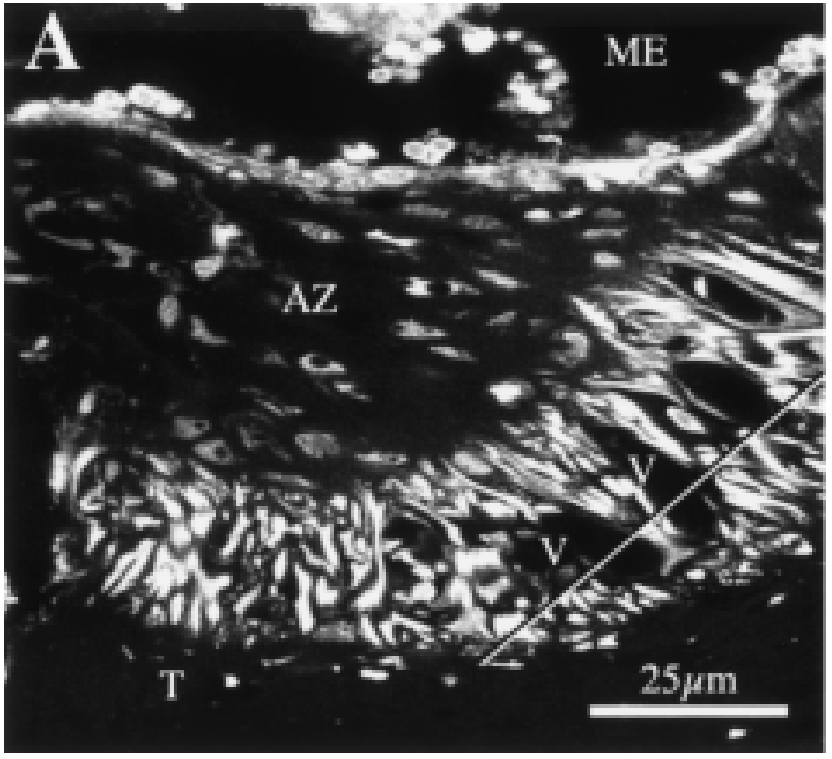

FIG. 4. Confocal microscopic images of the annulus fibrosus in a cross section (A) and in a section cut roughly parallel to the edge of the tympanic sulcus (B). A, P. parnellii.; B, P. quadridens. In A, the diagonal line shows the approximate plane of section for the image shown in B. The depth of field for these images was $0.5 \mu \mathrm{m}$ and the fluorescence was restricted to the smooth muscle cells in the

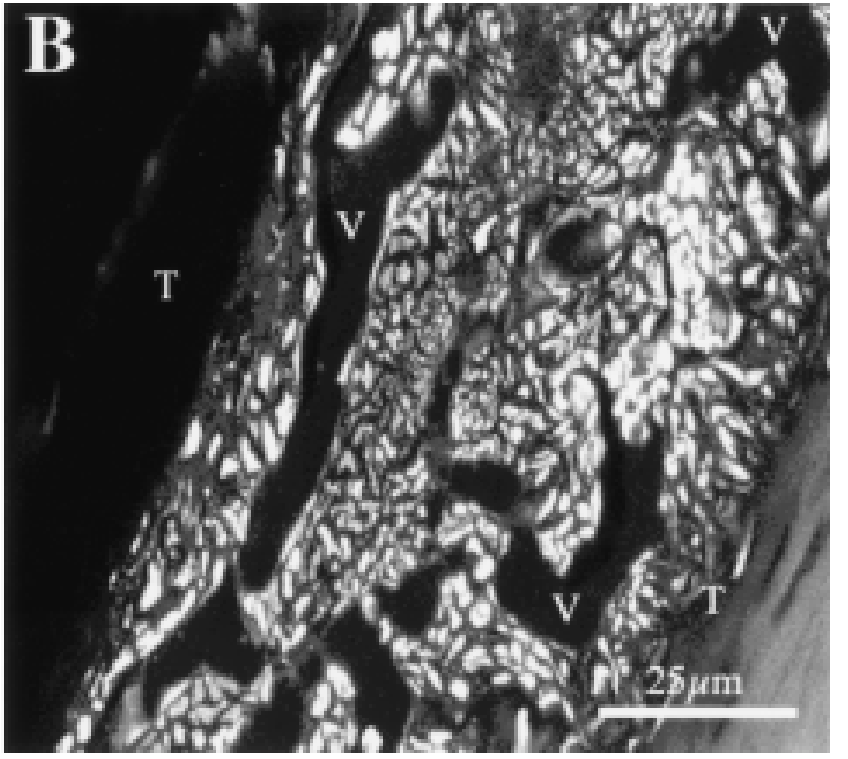

myovascular zone, scattered fibroblasts and the mucosal lining of the middle ear (ME). A few red blood cells within the vessels (V) and middle ear cavity are also apparent. In B, note the tortuous, interconnecting arrangement of the blood vessels $(\mathrm{V})$ and the complete filling of the intervascular spaces with the fluorescent smooth muscle tissue. The tympanic bone (T) is nonfluorescent.

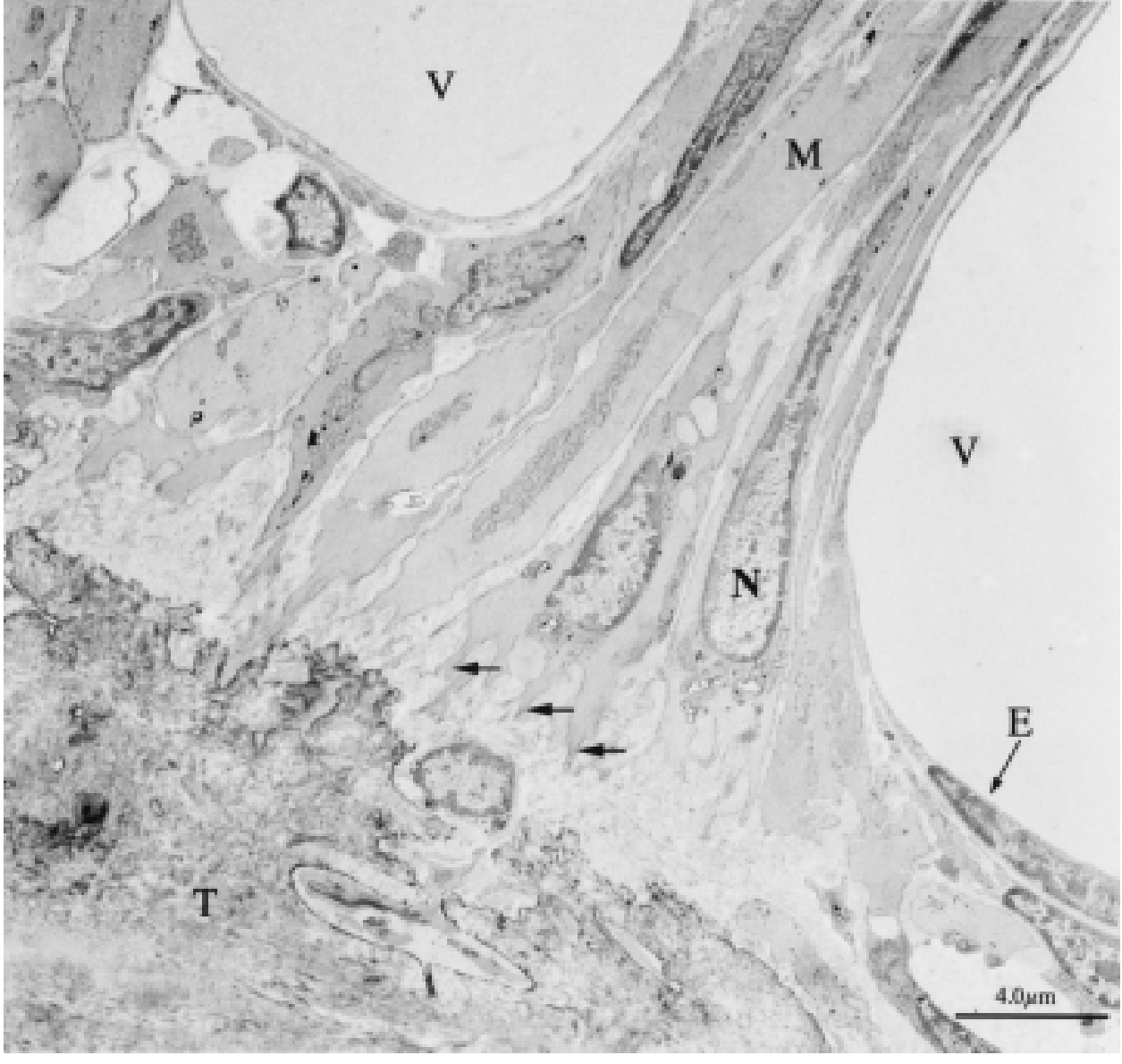

FIG. 5. TEM micrograph of the basal part of the myovascular zone (P. p. portoricensis). Note the elongated nucleus $(N)$ of one of the smooth muscle cells. A thin endothelial cell (E) lining the wall of one of the vessels $(\mathrm{V})$ is shown along with the interposed muscle tissue (M). Note that branches of the muscle fibers extend radially toward the tympanic bone $(\mathrm{T})$. Arrows, fimbriated ends of smooth muscle cells. 


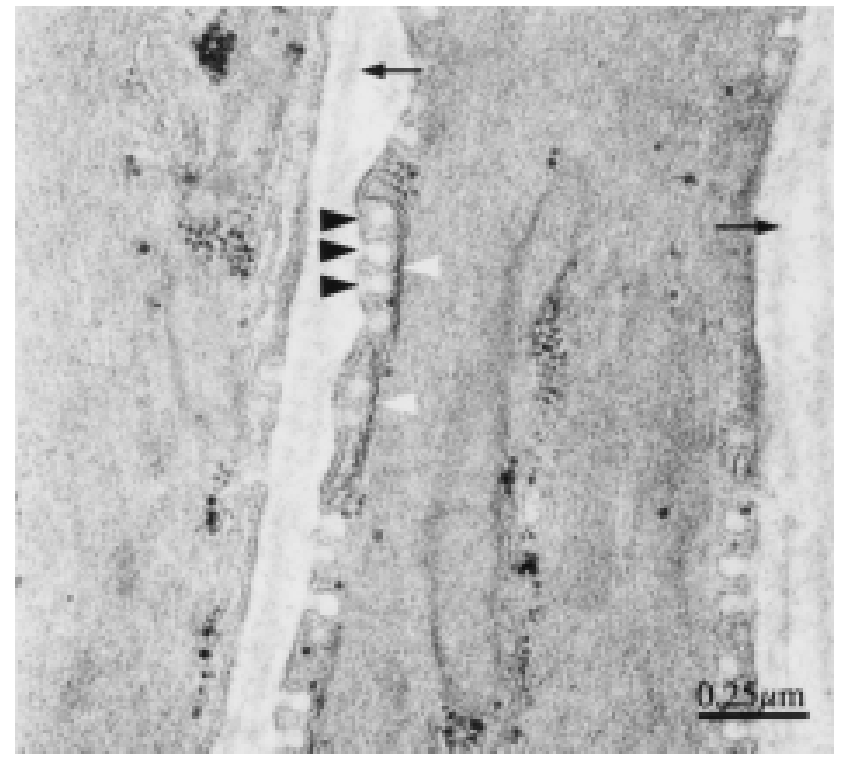

FIG. 6. Ultrastructural features of the bodies of smooth muscle cells in the central part of the myovascular zone (P. p. portoricensis). Note the basal lamina (arrows), the micropinocytotic vesicles aligned along the surface of the sarcolemma (black arrowheads), and electron dense bodies (white arrowheads) just deep to the vesicles. The latter represent the attachment points of myofilaments that appear as fine striations throughout the cytoplasm.

within a dense collagenous matrix. The curving base of the annulus conforms to the shallow concavity of the tympanic sulcus of the tympanic bone where the annulus is attached. Cross-sectional profiles of a series of relatively large blood vessels are a characteristic feature of the basal half, along with striations of radially arranged, interposed muscle fibers. This basal half will be referred to as the myovascular zone.

In cross sections of the annulus examined with confocal microscopy (Fig. 4A), the myovascular zone was well defined by the fluorescent images of the smooth muscle between the cross-sectional profiles of the blood vessels. By focusing through the tissue, the orientation, length and branching of many muscle fibers could be followed. The course and arrangement of the vessels in the myovascular zone were best seen in sections approximately parallel to the slightly curving surface of the tympanic sulcus (Fig. 4B). The vessels were tortuous and interconnected and had anastomotic connections with vessels running beneath the skin of the external acoustic meatus, the mucosal lining of the middle ear, capillaries in the apical zone and vessels in the tympanic bone.

Figure 5 shows a TEM micrograph through the basal half of the myovascular zone. Here the thin-walled nature of the vessels and the general appearance and orientation of the muscle fibers can be appreciated. The vessels are endothelial tubes with a wall thickness of $0.1-0.5 \mu \mathrm{m}$. Some of the adjacent muscle tissue is closely associated with the endothelium, but rather than encircling the vessels, as a tunic, the fibers and their branches extend radially toward the apical zone and basally toward the tympanic sulcus.

Smooth muscle in general has a number of characteristics (Burnstock, 1970; Gabella, 1981; Rhodin, 1974) and many of these features are illustrated in the TEM micrographs in Figures 5, 6, 7, 8, and 9. They include an elongated nucleus (Fig. 5), fine striations
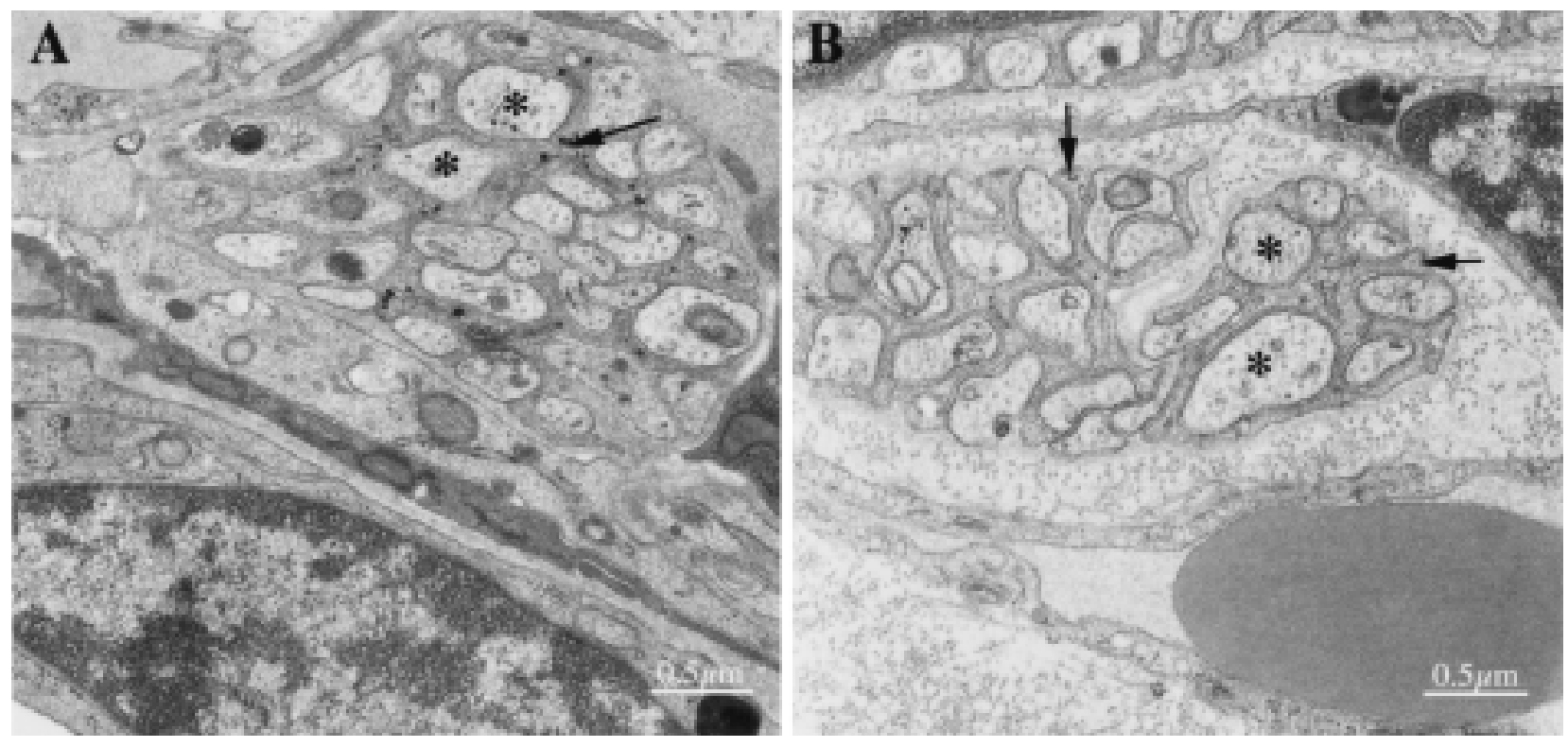

FIG. 7. TEM micrographs showing examples of cross sections of unmyelinated nerve fibers (asterisks) surounded by cytoplasmic infoldings of Schwann cells (arrows). These bundles were abundant in the MV zone and were often interposed between smooth muscle and the endothelial walls of vessels. An endothelial cell nucleus is evident in A. In B, the dark oval image (with scale bar) is a red blood cell in a capillary. 

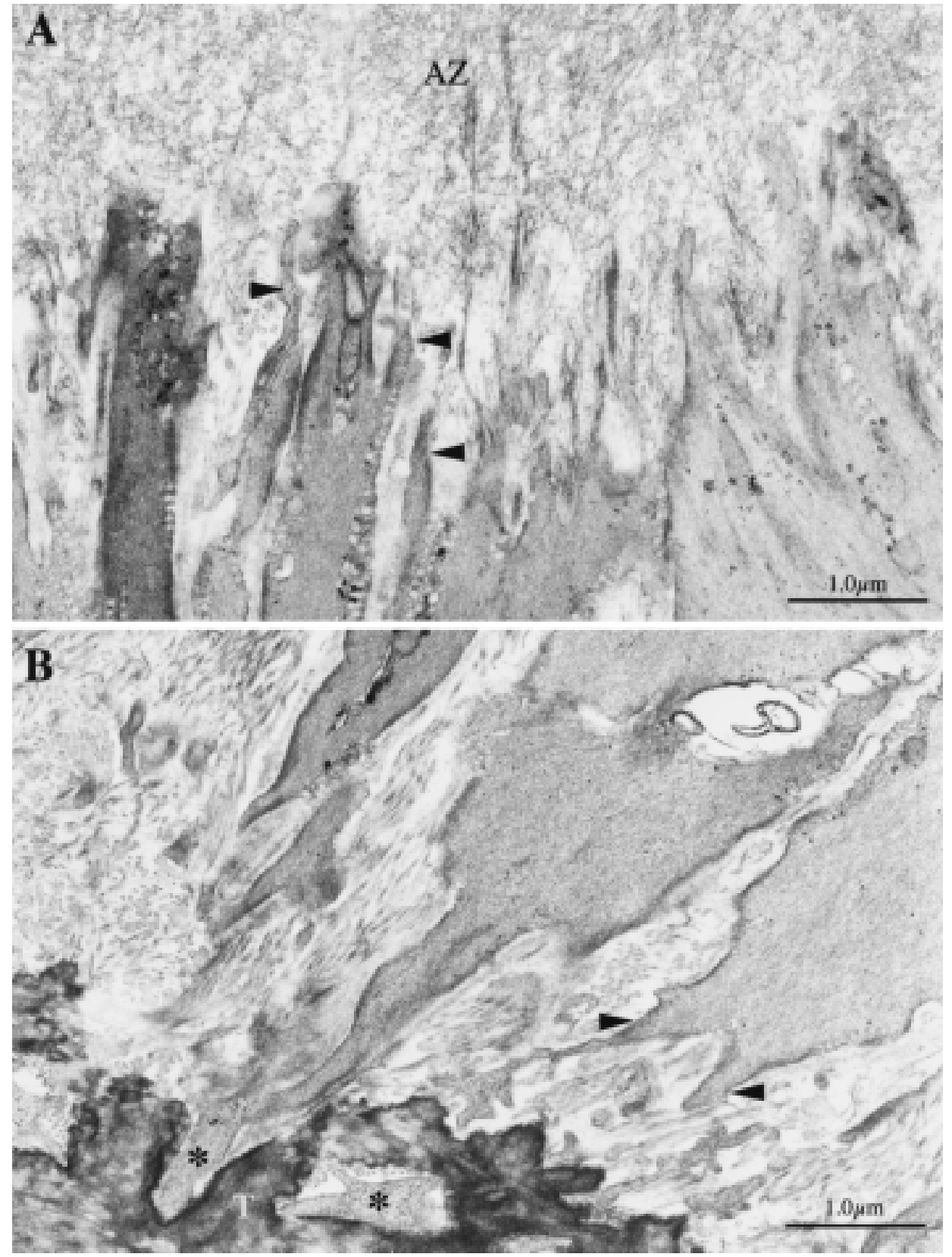

FIG. 8. TEM micrographs illustrating the fimbriated ends of smooth muscle cells and their association with the extracellular matrix (P. p. portoricensis). A shows muscle branches (arrows) at the junction of the myovascular and apical zones (AZ). Note the different electron density of the muscle branches (a common feature of smooth muscle) and the apparent attachment of the extracellular, radially arranged collagen fibers to the muscle. B shows branches (arrows) projecting toward the tympanic bone; asterisks mark the contact of muscle branches with bone $(\mathrm{T})$. which represent myofilaments (Fig. 6), elongated electron dense areas that are alpha-actinin associated attachment points for the myofilaments (Fig. 6), a basal lamina (Fig. 6), large numbers of micropinocytotic vesicles in or near the sarcolemma (for sequestering calcium) (Figs. 6 and 9A) and bundles of unmyelinated nerve fibers in the vicinity of the muscle fibers and blood vessels (Fig. 7). Also, commonly seen in smooth muscle are cells with different degrees of electron density (Fig. 8A).

Some features common to smooth muscle were not evident in our material. The cells, for example, are usually described as fusiform with a centrally located nucleus and tapered ends; those in the MV zone have elongated cell bodies and branched (fimbriated) ends (Fig. 5). Another feature often found in smooth muscle that was not apparent was cell to cell cohesion by gap junctions; in the MV zone the muscle cells and their processes always appeared to be separated by well defined intercellular spaces with basal laminae (Figs. 5 and 6).

The muscle cell branches and their association with radially oriented fibers of the connective tissue matrix are shown in Figures 5, 8, and 9. The branches that run apically (Fig. 8A) merge with the dense tissue of the apical zone and the branches at the basal end (Fig. 

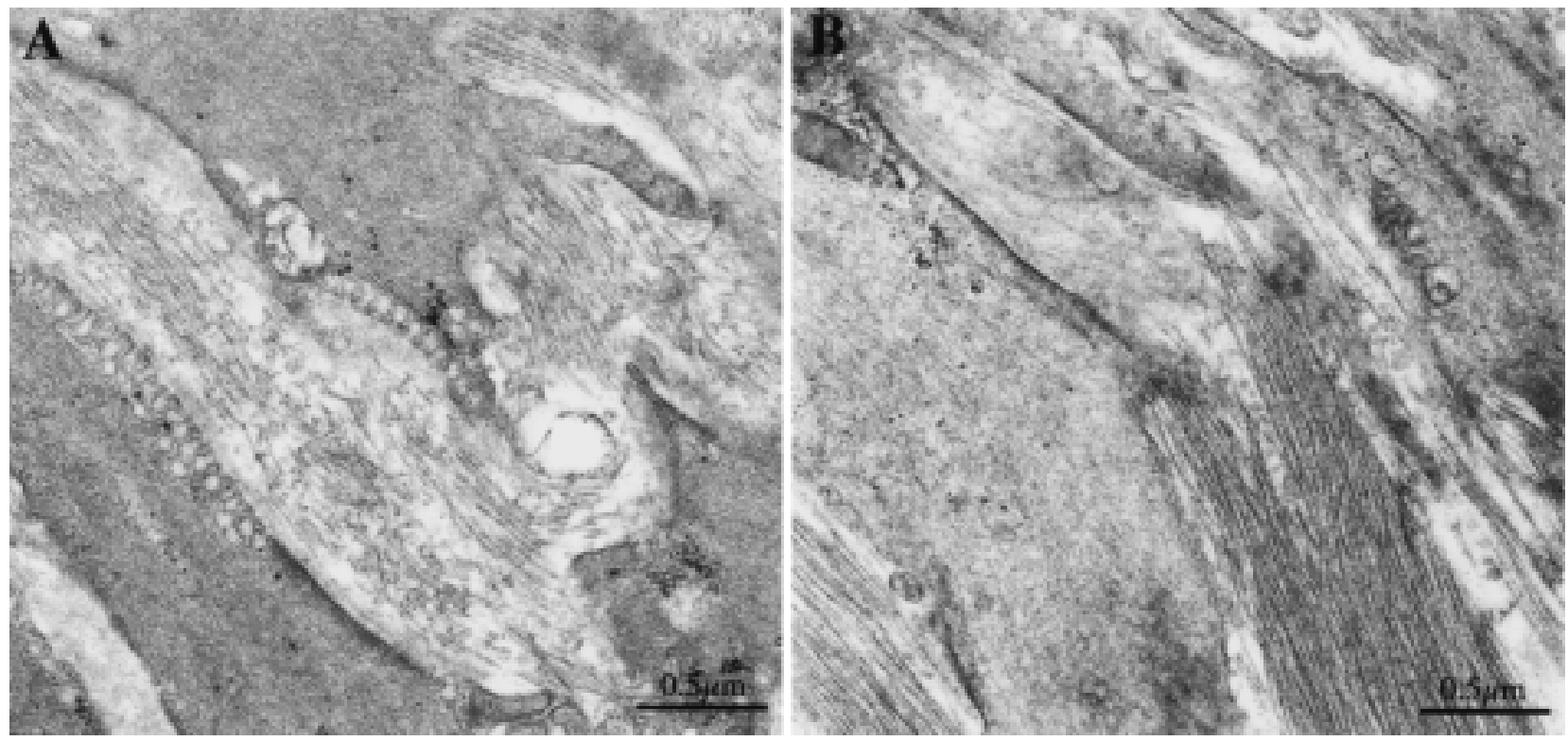

FIG. 9. TEM micrographs illustrating the parallel arrangement of extracellular collagen and muscle branches in the basal part of the MV zone (P. p. portoricensis). The collagen fibers in B are attached to the muscle at electron dense points.

8B) extend toward the tympanic sulcus. In both areas many collagen fibers were radially arranged and had the same orientation as the muscle branches.

The close association of the ends of the smooth muscle fibers with the extracellular matrix is illustrated in Figures 9 and 10. In relation to the elongated cell bodies, it was previously noted that the only extracellular matrix is the basal lamina (Fig. 6) which is composed of collagen fibers of small diameter. On the other hand, the fimbriated ends of the muscle are surrounded by larger, radially oriented collagen fibers that appear to merge with the basal lamina and/or the sarcolemma of the muscle. Where they merged there was increased electron density at the sarcolemma (Fig. 9). Figure 10 shows the insertion of collagen fibers directly into bony microprojections from the surface of the tympanic sulcus and also the direct contact of muscle cell processes with bone.

\section{DISCUSSION}

In this study we demonstrated that the connective tissue matrix of the pars tensa is continuous with a dense layer of connective tissue in the apical region of the annulus fibrosus. This region is in turn intimately associated with the fimbriated apical ends of a well-developed, closely packed array of radially oriented smooth muscle that is interposed between a network of thinwalled endothelial tubes. The occurrence of these vessels, numerous micropinocytotic vesicles in the sarcolemma of the muscle, and an abundance of unmyelinated nerve fibers indicates that the tympanic annulus is an active region with all the machinery necessary to tonically regulate tympanic membrane tension. The only other muscles capable of affecting tension are those of the middle ear and these are striated (twitch) muscles that have a limited ability to maintain tonic tension.

Kuijpers et al. (1999) have described what appears

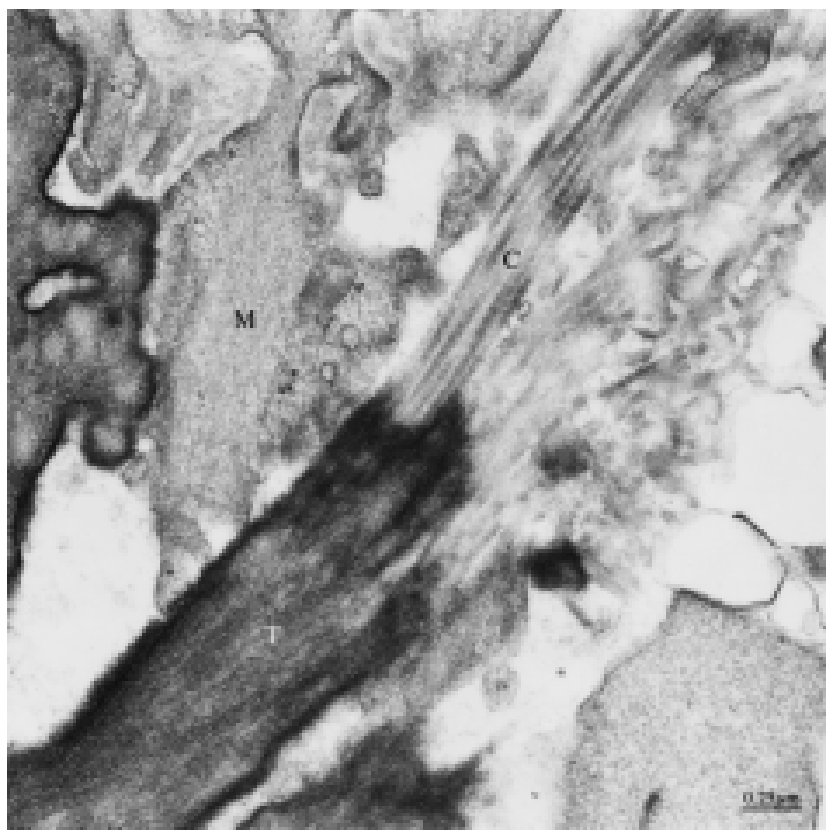

FIG. 10. TEM micrograph illustrating the insertion of collagen fibers, $C$, into microprojections of the surface of the tympanic sulcus and the attachment of muscle cell processes, $M$, to the bone, $T(P . p$. portoricensis). 
to be an analogous tension-generating (myofibrocyte) system in the annulus fibrosus of rats. Their studies indicated that there are three types of fibroblasts in the annulus and on the basis of immunostaining for smooth muscle actin, desmin, and vimentin, two of these types can be classified as myofibrocytes, a type of contractile element commonly found in scar tissue and other matrices where tension is encountered. The cells that they described in the annulus of the rat were clearly fibroblasts and not smooth muscle like that in the annulus of mustached bats. This was evinced by the absence of three distinguishing ultrastructural features of smooth muscle, all of which were evident in our material. These were the basal lamina, unmyelinated nerves and a sarcolemma with micropinocytotic vesicles. In addition, in the rat, the myofibrocytes had cell-to-cell contact (junctional complexes) and appeared to intermingle with radially oriented collagen. In the bat, the muscle cells were closely packed, did not appear to be linked by junctional complexes and radially oriented fibers were attached to both ends of the muscle. Another difference between the annulus in the bat and rat is the apparent absence or poor development of the network of endothelial tubes that lies in close association with the muscle fibers. The myovascular zone in the mustached bats appears to represent a much more specialized system than in the rat. It is interesting to note, however, that the areas with contractile elements are in the same location in both species.

Kuijpers et al. (1999) suggested that the myofibrocytes in rats might be antagonistic to the inward pulling tensor tympani muscle. In our opinion, a contractile annulus could have a number of functions: it could augment the tension created by the tensor tympani, it could restore the membrane to a resting tension, or it could adjust the resting tension to best suit the acoustic environment. It is also possible that the smooth muscle cell contractions could affect the blood vessels in the myovascular zone but they appear poorly arranged to perform this function.

Whether smooth muscle is a common feature of the annulus in other bats or other mammals needs to be determined. Preliminary studies indicate that similar smooth muscle arrays occur in other species of bats, rodents, and some insectivores (Cannon, Henson and Henson, unpublished data). Thus, it does not seem warranted to associate this muscle and the tension it could create with a specialization for echolocation, sharp tuning or living in an environment with high levels of background noise.

This is only the second study that we know of to deal with the ultrastructural features of the attachment of the tympanic membrane to the tympanic ring and thus comparative studies are needed to evaluate this system more fully. Ultrastructural studies are currently being carried out on a variety of mammals and physiological studies have been initiated to study the activity and function of the system.

\section{ACKNOWLEDGMENTS}

A special acknowledgment is due to Dr. Armando RodríguezDurán for assistance in collecting bats in Puerto Rico; without his help it would not have been possible and we thank him very much. We would also like to thank Ms. V.J. Madden and Dr. R.L. Bagnell for expert technical assistance with all aspects of the microscopy. This work was supported by USPHS grant NIDCD DC 00114.

\section{REFERENCES}

BAIRD IL, Henson OW. Sectioning and staining whole heads of small animals. Stain Technol. 36:173-175, 1961.

BuRnSTOCK G. Structure of smooth muscle and its innervation. Bülbring E, Brading AF, Jones AW, Tomita T. Smooth Muscle, Williams \& Wilkins, Baltimore, 1970, 1-69.

GABELLA G. Structure of smooth muscle. BÜLBRING E, BRADING AF, Jones AW, TOMita T. Smooth Muscle: an assessment of current knowledge. University of Texas Press, Austin, 1981, 1-46.

HeNSON OW. Some morphological and functional aspects of certain structures of the middle ear in bats and insectivores. Univ. Kans. Sci. Bull. 42, 3:131-255, 1961.

Henson OW. Comparative anatomy of the middle ear. KeIdel WD, NefF WD. Handbook of Sensory Physiology Vol. V/1. SpringerVerlag, Berlin, 1974, 39-110.

Kuijpers W, Peters TA, Tonnaer ELGM. Nature of the tympanic membrane insertion into the tympanic bone of the rat. Hearing Res. 128:80-88, 1999

Lim DJ. Structure and function of the tympanic membrane: a review. Acta Oto-rhino-laryngol. Belg. 49:101-115, 1995.

MAdDEN VJ, Henson, MM. Rapid decalcification of temporal bones with preservation of ultrastructure. Hearing Res. 111:76-84, 1997.

RHODIN JAG. Histology: a text and atlas. Oxford University Press, New York, 1974. 